\title{
Patient Perceptions of Electronic Medical Record Use by Faculty and Resident Physicians: A Mixed Methods Study
}

\author{
Wei Wei Lee, MD, MPH' , Maria A. Alkureishi, MD, FAAP², Obioma Ukabiala, BA ${ }^{3}$, \\ Laura Ruth Venable, BS ', Samantha S. Ngooi, MPP', Daina D. Staisiunas, JD', \\ Kristen E. Wroblewski, MS', and Vineet M. Arora, MD, MAPP ${ }^{7}$
}

\begin{abstract}
'Department of Medicine, University of Chicago, Chicago, IL, USA; ${ }^{2}$ Department of Pediatrics, University of Chicago, Chicago, IL, USA; ${ }^{3}$ Pritzker School of Medicine, University of Chicago, Chicago, IL, USA; ${ }^{4}$ Department of Public Health Sciences, University of Chicago, Chicago, IL, USA.
\end{abstract}

\begin{abstract}
BACKGROUND: While concerns remain regarding Electronic Medical Records (EMR) use impeding doctor-patient communication, resident and faculty patient perspectives post-widespread EMR adoption remain largely unexplored.
\end{abstract}

OBJECTIVE: We aimed to describe patient perspectives of outpatient resident and faculty EMR use and identify positive and negative EMR use examples to promote optimal utilization.

DESIGN: This was a prospective mixed-methods study.

PARTICIPANTS: Internal medicine faculty and resident patients at the University of Chicago's primary care clinic participated in the study.

APPROACH: In 2013, one year after EMR implementation, telephone interviews were conducted with patients using open-ended and Likert style questions to elicit positive and negative perceptions of EMR use by physicians. Interview transcripts were analyzed qualitatively to develop a coding classification. Satisfaction with physician EMR use was examined using bivariate statistics.

RESULTS: In total, 108 interviews were completed and analyzed. Two major themes were noted: (1) Clinical Functions of EMR and (2) Communication Functions of EMR; as well as six subthemes: (1a) Clinical Care (i.e., clinical efficiency), (1b) Documentation (i.e., proper record keeping and access), (1c) Information Access, (1d) Educational Resource, (2a) Patient Engagement and (2b) Physical Focus (i.e., body positioning). Overall, $85 \%$ (979/1154) of patient perceptions of EMR use were positive, with the majority within the "Clinical Care" subtheme $(n=218)$. Of negative perceptions, $66 \%(115 / 175)$ related to the "Communication Functions" theme, and the majority of those related to the "Physical Focus" subtheme $(n=71)$. The majority of patients (90\%, 95/106) were satisfied with physician EMR use: $59 \%$ (63/107) reported the computer had a positive effect on their relationship and only $7 \%(8 / 108)$ reported the EMR made it harder to talk with their doctors.

CONCLUSIONS: Despite concerns regarding EMRs impeding doctor-patient communication, patients reported

Electronic supplementary material The online version of this article (doi:10.1007/s11606-016-3774-3) contains supplementary material, which is available to authorized users.

Received August 3, 2015

Revised January 27, 2016

Accepted June 8, 2016

Published online July 11, 2016 largely positive perceptions of the EMR with many patients reporting high levels of satisfaction. Future work should focus on improving doctors "physical focus" when using the EMR to redirect towards the patient.

KEY WORDS: electronic health records; patient-centered care; physician patient relations; communication skills.

J Gen Intern Med 31(11):1315-22

DOI: $10.1007 /$ s11606-016-3774-3

(C) Society of General Internal Medicine 2016

\section{BACKGROUND}

In the wake of federal mandates to promote electronic medical record (EMR) adoption as part of the Affordable Care Act (ACA), EMR use in clinical care has accelerated greatly since 2010. ${ }^{1,2}$ The ACA also calls for an emphasis on patientcentered care, particularly since research has shown patientcentered communication strategies can improve patient satisfaction and clinical outcomes, including medication adherence and acute care utilization. ${ }^{3-5}$ These dual directives translate into the need for physicians to be both clinically efficient with the EMR while maintaining meaningful interactions with patients. Few physicians receive formal training on how to practically achieve this, which may unfortunately result in physicians who pay more attention to the "iPatient" or virtual patient described by Dr. Abraham Verghese, who exists only on the computer screen, while the real patient in the exam room is neglected and alone. ${ }^{6}$

We conducted a systematic literature review on the effect of the EMR on the patient-physician relationship and communication. ${ }^{7}$ While observational studies identified behaviors that researchers believe promoted communication (i.e., clarification of information, facilitation of discussion), ${ }^{8-11}$ potentially negative behaviors (i.e., increased speech interruption and unsuccessful physician multitasking $)^{12-16}$ have been noted as well. $^{7}$ A limitation of most of these studies however is their lack of correlation between researcher-observed behaviors and the patient's own experience.

In order to provide patient-centered care in the setting of EMR use, it is crucial to elicit and understand the patient's perspective. In our review of studies examining the impact of EMR use on face-to-face communication and the patient- 
provider relationship, only six patient-perception studies have been published since 2012. ${ }^{7}$ While most demonstrated a lack of change in the patient-provider relationship and high satisfaction with EMR use, concerns such as provider distraction by the computer and changes in non-verbal aspects of communication such as eye contact and long periods of silence were raised. ${ }^{17-23}$

In addition, most studies were conducted prior to the 2013 Meaningful Use ACA mandates and rapid EMR adoption. ${ }^{7}$ As more patients encounter their physicians using EMRs in the exam room after ACA implementation, they may be better primed to share their experiences on this timely issue. Furthermore, study limitations including small physician sample size without specific analysis of resident patient perspectives, exclusive use of closed-ended patient surveys, and a paucity of recent US primary care studies make generalizability to a current US academic primary care setting difficult.

Understanding the discrepancy between objective evidence of potentially negative EMR-related communication behaviors and unchanged patient satisfaction requires a rigorous exploration of patient perspectives. A mixed methods approach, utilizing qualitative analysis of patient interviews and quantitative analysis of patient satisfaction, would allow better understanding of the patients' raw experiences with EMR use.

To address these gaps, we set out to examine and compare patient perspectives and satisfaction with resident and faculty clinic EMR use in the post-Meaningful Use era. Our study adds to this literature by using a mixed methods approach, targeting a larger patient sample and examining patient perceptions of both faculty and resident physicians. We aimed to identify patients' positive and negative experiences with the EMR, and elicit examples of exemplary and ineffective EMR use to optimize patient-centered utilization. Ultimately, these findings can be used to design a patient-centered EMR use curriculum to optimize the patient experience.

\section{METHODS}

\section{Setting and Study Design}

This study occurred at a single, academic primary care clinic, with 32 general medicine faculty and approximately 30 Internal Medicine residents per class. All patients included were seen in clinic between August and October 2013, approximately 1 year after ambulatory EMR implementation. Using a random number generator, patients were selected for telephone interviews conducted between December 2013 and August 2014.

\section{EMR Implementation}

The University of Chicago implemented the EMR system into ambulatory practices in May 2012. Prior to implementation, faculty and residents participated in a minimum of $8 \mathrm{~h}$ of required EMR training focusing on technical aspects related to patient care (i.e., order entry, note writing). No training on communication skills or how to optimize EMR use with patients in the exam room was conducted.

\section{Data Collection}

Internal medicine faculty and residents gave written consent to allow us to contact their patients for participation in telephone interviews. Patients were called by one of three trained Research Assistants (RAs) (DS, LV, or OU) and invited to participate in the interview between December 2013 and August 2014. RAs were non-physicians and not previously known to research participants. Patients were consented to participate using a formal oral consent script. Inclusion criteria included ability to consent and speak English. Interviews were recorded, professionally transcribed from digital telephone audio recording and anonymized by assigning each participant a code number. This study was approved by the University of Chicago Institutional Review Board.

Based on our previously published literature review on the impact of EMR use on patient-doctor communication, we designed our interview script to included nine Likert-style questions and three yes or no questions with subsequent open-ended follow-up questions to elicit positive and negative experiences with physician computer use in the exam room (Online Appendix 1). ${ }^{7,24,25}$ For the Likert scale questions, patients rated their level of agreement on a fivepoint scale from "never" to "always" and from "strongly disagree" to "strongly agree" for specific statements about satisfaction and impact of computer use on patient-doctor communication.

The script was designed to be easily understood by patients (i.e., substituted 'computer use' for 'EMR use'). Interviews were conducted by the same three RAs (DS, LV, and OU). The interview was semi-structured with open-ended questions to elicit positive and negative experiences with physician computer use (i.e., "Do you like it when your doctor uses the computer when you see him/her in the clinic?"). Semistructured probing questions were also included in the script and improvised by the interviewers to prompt patient comments (i.e., "What does your doctor do with the computer that you don't like? Can you give me examples?").

Based on previous telephone interview studies at the University of Chicago with the same patient population and similar interview techniques, we estimated 100 patient interviews were sufficient to achieve theme saturation. Theme saturation was achieved when no new themes were identified despite analysis of additional transcripts. ${ }^{26}$

\section{Data Analysis}

For the mixed methods analysis of our structured interview, we analyzed patient responses to the open-ended questions and Likert scale questions. Qualitative analysis of transcripts was completed using the ATLAS.ti 7.1 (Berlin, Germany) 
software program. Two physician investigators (WL and MA) and one RA (LV) coded $20 \%$ of the interview transcripts to establish a coding scheme. ${ }^{26,27}$ Using an iterative process of listening and discussing, the investigators met at regular intervals to resolve discrepancies by consensus. An additional $10 \%$ of transcripts were coded independently by two physician investigators (WL and MA) and one trained RA (OU) to establish inter-rater reliability (IRR) using the CAT (coding analysis toolkit) software (@ 2007-2015, Pittsburg, PA USA) to calculate a three rater Cohen's kappa score of 0.63 , indicating a substantial and acceptable level of agreement. ${ }^{28}$ After IRR was achieved, the remaining transcripts were coded by the RA (OU) utilizing the coding scheme. To ensure validity of our results, another author (WL) reviewed the transcripts and all coded quotations to ensure accuracy.

Descriptive statistics of patient Likert scale responses were examined and data was dichotomized for analysis (i.e., "agree" was defined as a Likert response of agree or strongly agree). Patient satisfaction data were examined quantitatively using Stata 13.0 (StataCorp., College Station, TX). Wilcoxon rank sum, Pearson's chi-squared, and Fisher's exact tests were utilized as appropriate to examine associations between patient age $(<60$ versus $60+$ years old) and satisfaction measures and to test for differences between resident and faculty patients' responses.

Our paper conforms to the Standards for Reporting Qualitative Research (SRQR) standards. ${ }^{29}$ Reflexivity was maintained by the research team by discussing and challenging established assumptions at regularly scheduled research meetings. One physician investigator (WL) conducted member checks with five patient participants to ensure validity of the analysis.

\section{RESULTS}

A total of 9348 patients seen by 28 faculty and 81 resident internal medicine physicians between August and October 2013 were identified and 384 were randomly selected to include on our call list. After attempting to contact all 384 patients up to six times, we reached $193(50 \%, 193 / 384)$ on the phone (i.e., excluded unable to consent, non-English speaking, did not answer, deceased etc.; Figure 1). Of these patients, $110(57 \%, 110 / 193)$ consented and $108(98 \%, 108 /$ 110) patient interviews were completed.

Of 108 patients interviewed, $51(47 \%, 51 / 108)$ were seen by 28 unique faculty members and $57(53 \%, 57 / 108)$ were seen by 45 unique residents. Average patient age was 61 (range 21-92), $67 \%(72 / 108)$ were female and $67 \%(72 /$ 108) were African American. Patients seen by faculty were older than resident patients (mean age 66 vs. 56, $p<0.05$ ), but had similar proportions of female [67\% (34/51) vs. $67 \%$ (38/ 57), $p=1.0]$ and African American patients [65 \% (33/51) vs. $68 \%(39 / 57), p=0.68]$.

Non-responders did not differ in average age [mean 57.8 (range 20-99), $p>0.05$ ] and had similar proportions of female
$(61 \%, p>0.05)$ and African-American patients (67 \%, $p>$ $0.05)$ compared to responders.

\section{Qualitative analysis: Positive and Negative Experiences Related to Physician Computer Use}

Patients identified 21 pairs of positive and negative experiences related to physician computer use. Positive and negative experiences were categorized into two major themes: (1) Clinical Functions of EMR and (2) Communication Functions of EMR. Within the two major themes, we identified six subthemes: (1a) Clinical Care (i.e., clinical efficiency), (1b) Documentation (i.e., proper record keeping and access), (1c) Information Access, (1d) Educational Resource, (2a) Patient Engagement and (2b) Physical Focus (i.e., body positioning) (Online Appendix 2). Representative patient quotes are provided in Table 1.

Overall, $85 \%$ (979/1154) of total codes reflected positive perceptions of EMR use with the majority of these falling under the "Clinical Care" subtheme (218). Patients liked that the EMR increased their doctor's clinical efficiency, allowed them to review other physicians' notes and promoted teamwork and communication between doctors; "They refer to each other's notes and communicate about what's going on with me. It makes me comfortable with the care I'm getting." The second most commonly reported positive experience with the EMR related to the "Patient Engagement" subtheme (212). Patients liked that their doctors used the EMR to promote patient engagement and discussion and used visuals (i.e., pictures and graphs) to explain their care. They also liked when their doctors were transparent in their use of the EMR, explaining what they were doing on the computer, resulting in a perception of increased quality of care.

Patients also identified negative experiences related to EMR use in the clinic setting. While only $15 \%(175 / 1154)$ of total patient perceptions were negative, the most common were within the "Physical Focus" subtheme (71), with patients reporting perceptions of unbalanced focus (i.e., physicians more focused on computer than patient) (45), poor eye contact (12) and poor screen positioning (12); "I just want my doctor's undivided attention ...the computer takes them away from focusing on you." The second-most reported negative experience related to "Patient Engagement" (44), and patients disliked when physicians did not use the computer to facilitate discussion (19) or failed to explain what they were doing on the computer (7), resulting in perceptions of decreased quality of care (15). Member checks with five patients confirmed the positive and negative perceptions described above.

\section{Quantitative Analysis: Patient Satisfaction Data}

While most patients $(69 \%, 75 / 108)$ liked when their doctors used the computer during the visit, nearly one-third (29\%, 31/ 108 ) were ambivalent and had variable experiences. Similarly, while $59 \%(63 / 107)$ reported that computer use had a positive 


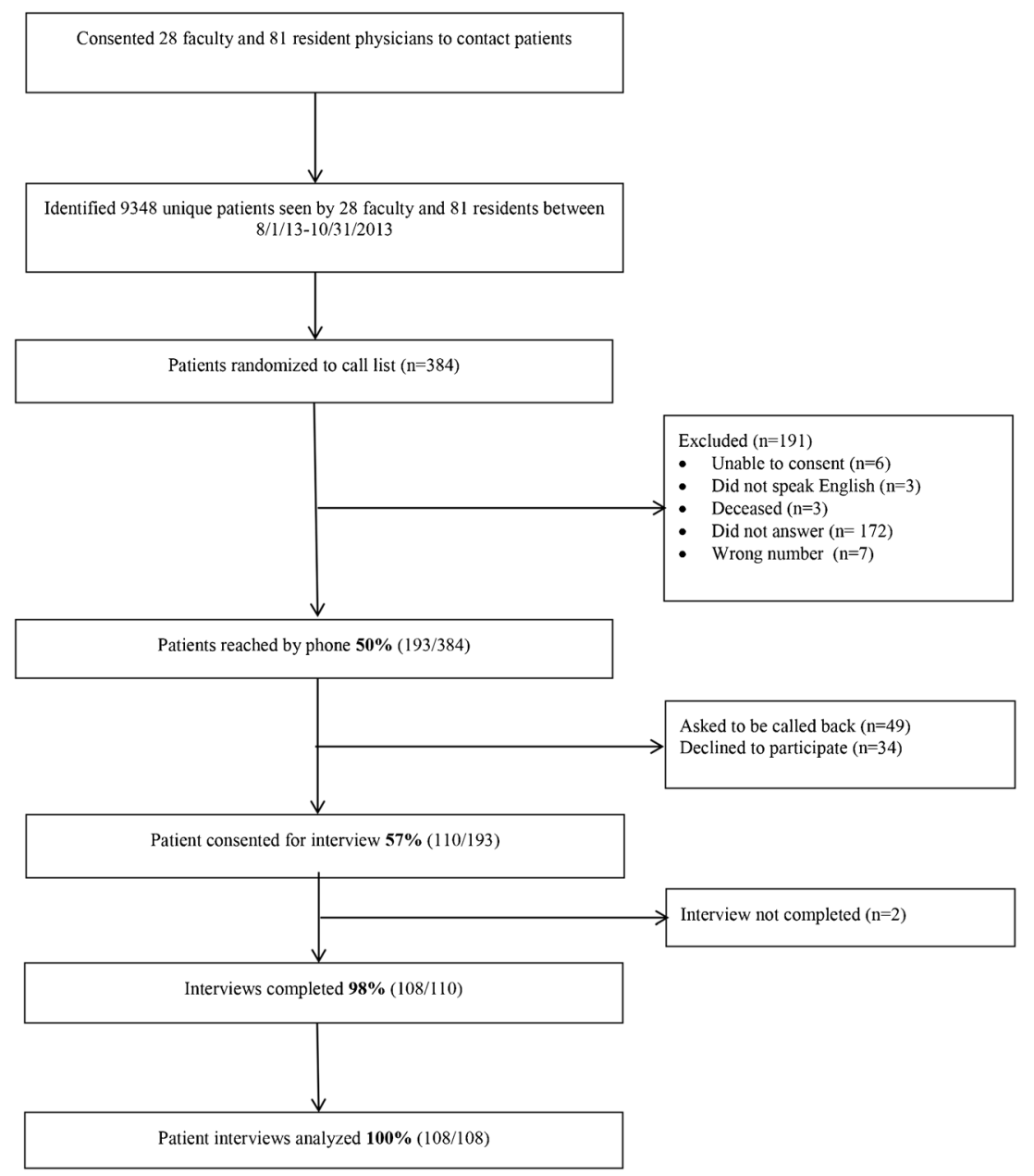

Figure 1. Patient enrollment for telephone interviews.

effect on their relationship with their doctor, $39 \%(42 / 107)$ were unsure or found variability in the experience (Table 2).

Overall, the large majority of patients $(90 \%, 95 / 106)$ were satisfied with physicians' computer use in the clinic setting. Most patients reported their doctor's computer use helped them to better understand medical conditions and treatments $(81 \%, 85 / 105)$ and made it easier for them to communicate during the visit $(75 \%, 78 / 104)$. The majority of patients $(88 \%, 90 / 102)$ reported their doctors stopped using the computer and were fully focused on them when they discussed sensitive topics. Only $8 \%(8 / 106)$ of patients agreed that the computer was disruptive and prevented their doctor from focusing on them.

Patients also reported negative perceptions of computer use. Less than half of patients $(47 \%, 47 / 100)$ reported that their doctor positioned the screen to allow them to see the screen and were concerned with this lack of transparency. Moreover, while only $8 \%(8 / 103)$ of patients cited poor eye contact with their physicians, $26 \%(27 / 103)$ reported that they at least sometimes felt their doctors looked more at the screen than at them.

In comparing faculty and resident patient responses to the satisfaction questionnaire, the only significant difference was for overall satisfaction with the doctor's use of the computer in the clinic room, with faculty patients reporting higher satisfaction when compared with resident patients (mean [SD] 4.62 [0.60] vs. 4.27 [0.86], $p=0.02)$. When comparing overall satisfaction of patients $<60$ years old $(n=41)$ to those $\geq 60$ $(n=66)$, there was no significant difference in their responses ( $p>0.05$ for all).

\section{DISCUSSION}

To our knowledge, this is the first US-based study using mixed methods to systematically analyze both resident and faculty patient interviews exploring experiences with clinical EMR use in the post-Meaningful Use era. By exploring patient experiences one year after EMR implementation, several positive and negative experiences related to effective computer use in the clinic setting were identified. The majority of patients were satisfied with their physicians' overall use of the computer. Patients reported that when used well, the EMR could be effectively used as a tool to facilitate communication and promote better understanding of their medical problems and treatment plans. Moreover, patients felt computer use 
Table 1. Themes and Representative Quotations of Positive and Negative Patient Experiences with Physician Computer Use in the Exam Room

Major Theme (1) Clinical Functions of EMR (667)

Subtheme (1a) Clinical Care (250)

Codes $(n)$

1. Clinical Efficiency (148)

2. Teamwork (57)

3. Chart review (40)

4. Coordination of Care (5)

Subtheme (1b) Documentation (195)

5. Medical history (77)

6. Records Information (69)

7. Conscientious (i.e., follow-up labs, vaccines, etc.) (37)

8. Chart review (12)

Subtheme (1c) Information Access (176)

9. Accessibility of information (164)

10. Suitable information access (12)

Subtheme (1d) Educational Resource (46)

11. Medical Questions (36)

12. Patient Education (10)
Example of Positive Code $(n)$

"It makes the visit go smoother...they take notes and pull up my record... they don't have to flip through a huge chart." $110(n=126)$

"They refer to each other's notes and communicate about what's going on with me. It makes me comfortable with the care I'm getting." $117(n=49)$ "He can go back and look at important test results- he had it at his fingertips." $177(n=39)$

"He could tell me right away that I had an upcoming appointment with my specialist." $79(n=4)$

"They're asking me questions to verify and make sure everything is correct." $122(n=73)$

"I like that he repeats and recaps what we talked about while he types it in. I am confident he captured what we discussed." $81(n=59)$

"He used the computer to see what other tests I needed...if I haven't had a test done in a certain amount of time he lets me know." $120(n=35)$ "They've got a quick run through of my records and a better handle before they walk in and see me." $155(n=11)$

"It seems more professional...with my records online they have easier access so they actually look at it." $103(n=163)$ "It doesn't bother me that the nurses can see my file because that's my doctor's team...I trust my doctor." $143(n=6)$

"I had a question ....and he went online and looked it up and gave me the answer." $171(n=34)$

"We talked about a condition he thought I had ... he used the computer, pulled up information and printed it out for me." $103(n=8)$
Example of Negative Code $(n)$

"The doctor spends too much time recording and making notes - I don't know what else they're doing." $86(n=22)$

"With technology all these doctors are connected, but it doesn't seem like there's much communication going, my doctors don't talk to each other." $121(n=8)$

"I know there are CT scans, but it seems like they don't know it's in the chart." $121(n=1)$

"The doctors don't help to schedule future appointments." $129(n=1)$

"There are inaccuracies in the computer and at each clinic I go to, they're not always updating it." $119(n=4)$

"They have to enter codes...it takes a lot of time and takes away from dealing face to face with patients." $177(n=10)$

"The doctor forgot to give me a flu shot. When I went back, they were out." $101(n=2)$

"They don't even know what the record states when they come in the room." $84(n=1)$

"Sometimes the computer doesn't work and they can't access my records." $113(n=1)$

"Regardless of who printed out your paperwork, at checkout it shows everything that was going on...It was an invasion of privacy." $151(n=6)$

"He said give me five minutes and I'll look it up on the computer... But he didn't find anything." $120(n=2)$

"If you're going to use a computer, look up my diagnosis and explain to me the type of cancer that I have." $84(n=2)$

\section{Major Theme (2) Communication Functions of EMR (487)}

Subtheme (2a) Patient Engagement (256)

1. Facilitate patient discussion (123)

2. Quality of care (51)

3. Use of visuals (charts, graphics, etc.) (43)

4. Explain what they are doing on computer (22)

5. Patient Questions (17)
"We talk and look at results together in the computer... we had an intelligent conversation about my progress." $121(n=104)$

"He's telling you all along... what he's doing. This improves the quality of my care." $75(n=36)$

"They used diagrams and pictures in the computer to explain my medical condition, they also printed it out so I could take it home to my family." $117(n=41)$

"He puts something in the computer and then he'll explain to me what he's putting in." $118(n=15)$

"I might have questions for my doctor that I forget, but going through my record together causes me to remember my questions." $80(n=16)$
"They need to be more responsive to patients, ask patients do you have questions. That doesn't occur very often." $123(n=19)$

"You can't just look in the computer and say everything was fine. You need to check me out. You're not making a relationship with the computer. You're making a relationship with your patient." $84(n=15)$

"If he used some graphs or charts, I might say that was effective communication." $95(n=2)$

"They're looking at the computer constantly when I'm in the room. I know they're not on Facebook but I don't know what they are doing." $84(n=7)$ "They read what's on the screen and have a few seconds to test you with the stethoscope and then rush off. Sometimes you forgot to say something but that's it. Your time is gone." $152(n=1)$ 
Subtheme (2b) Physical Focus (231)

6. Balance of Focus (136)

7. Eye contact (44)

8. Screen positioning (41)

9. Body Positioning (10)
My doctor turns to the computer to look up something, then turns immediately back to me. It doesn't get in the way of anything. It only helps." $95(n=91)$

"He'll make eye contact with me more than the computer. He's not ignoring me."

$118(n=32)$

"She shows me the screen and points out things, so I can see what she sees and then she'll discuss it." $160(n=29)$

"When I was talking about something important, the doctor faced me, stopped

looking at the computer and listened to me." $155(n=8)$
"I just want my doctor's undivided attention, so I can tell him what's going on...the computer takes them away from focusing on you." 85 (45)

"He was talking and looking at the computer the whole time. I don't get the human connection." $84(n=12)$

"I never see the screen with the doctors. The computer's not facing me." $85(n=12)$

"Some come in and stare right at the screen, hunkered down...their back to you, it's not patient friendly." $8(n=2)$

Patient comments were edited for readability

allowed doctors to increase clinical efficiency, allowed easy access to their health information, promoted accurate documentation in real time and enhanced teamwork with other physicians.

Patients also reported several negative perceptions related to computer use in the exam room. While our study found higher rates of screen sharing than those reported in the literature, patients still expressed frustration when physicians were not transparent with EMR use by not allowing them to see the screen or explaining what they were doing. ${ }^{30}$ Patients also cited poor screen positioning, body positioning and eye contact as factors contributing to perceptions of decreased quality of care and unbalanced focus, with the physician appearing more focused on the computer than the patient. Furthermore, patients expressed dissatisfaction with physicians who did not engage the EMR to discuss their health or provide education.

Interestingly, compared with resident patients, faculty patients reported higher satisfaction on only one question, which assessed patient satisfaction with their doctor's overall use of computers; there was no difference between the two groups on questions related to screen sharing, eye contact or other computer-related experiences. It is important to note patient age did not have an impact on how patients perceived computer use in the clinic setting, thus assuaging concerns that older patients may be more technology-averse than younger patients.

Our findings show a third of patients remain ambivalent about their physician's computer use, with many of these negative experiences centered on communication related issues. These negative experiences can be overcome by training physicians to integrate patient-centered communication strategies while using the EMR in clinical practice. Unfortunately, few physicians are formally taught how to use the EMR in a patient-centered way. ${ }^{31-36}$

This study has implications for future design of communications curricula as well as the content of required EMR training at healthcare facilities. Our findings and best practices could be used to guide the development of a patient-centered EMR use curriculum for faculty and trainees. ${ }^{32}$ Such a curriculum might include training on using the EMR to promote teamwork amongst care physicians, maximizing patient engagement and education by reviewing health information

Table 2. Patient-Reported Satisfaction with Physician Computer Use

\begin{tabular}{|c|c|c|c|}
\hline Question & Agree $^{a}$ & $\%$ & Mean (SD) \\
\hline $\begin{array}{l}\text { Do you like it when your doctor } \\
\text { uses the computer during the clinic visit? }\end{array}$ & $75 / 108^{\mathrm{b}}$ & 69 & \\
\hline When your doctor uses the computer, does it make it harder for you to talk to your doctor? & $8 / 108^{\mathrm{b}}$ & 7 & \\
\hline When your doctor uses the computer, does it have a positive effect on your relationship? & $63 / 107^{\mathrm{b}}$ & 59 & \\
\hline My doctor positions the computer so I can see the screen & $47 / 100$ & 47 & $3.25(1.53)$ \\
\hline My doctor looks at the computer screen more than he/she makes eye contact with me & $8 / 103$ & 8 & $1.89(1.03)$ \\
\hline $\begin{array}{l}\text { When I am talking about a sensitive topic, my doctor stops using the computer and pays full } \\
\text { attention to me }\end{array}$ & $90 / 102$ & 88 & $4.60(0.90)$ \\
\hline The computer is disruptive and prevents the doctor from focusing on me & $8 / 106$ & 8 & $1.72(1.05)$ \\
\hline $\begin{array}{l}\text { My doctor's use of the computer allows me to better understand my medical conditions and } \\
\text { treatment plans }\end{array}$ & $85 / 105$ & 81 & $4.10(1.14)$ \\
\hline My doctor's use of the computer makes it easier for us to communicate & $78 / 104$ & 75 & $4.04(1.21)$ \\
\hline My doctor's use of the computer strengthens our relationship & $70 / 107$ & 65 & $3.81(1.23)$ \\
\hline Overall, I am satisfied with my doctor's use of the computer in the clinic room & $95 / 106$ & 90 & $4.43(0.77)$ \\
\hline
\end{tabular}

${ }^{a}$ Proportion of patients answering $\geq 4$ on five-point scale unless otherwise indicated

${ }^{b}$ Proportion of patients answering Yes 
together, sharing the screen to promote transparency and optimizing eye contact and body positioning.

Currently, most physicians are required to attend EMR training sessions that focus on technical aspects of EMR use, but do not address patient-doctor communication. At our institution, we have integrated best practices for patientcentered EMR use into the required EMR training for all incoming residents and fellows. Given the focus on patient experience in healthcare, incorporating best practices of patient-centered EMR use into such training can improve the quality of the patient-doctor relationship.

There were several limitations of our study. First, it was a single institution study limiting generalizability of results. Second, it was difficult to reach patients on the phone and we were unable to interview all patients, which may result in nonresponse bias. In addition, we only solicited the views of primary care clinic patients who are more likely to have long standing relationships with their physicians, which may impact perceptions of computer use, and results may differ when interviewing specialty clinic patients. Lastly, social desirability bias may have impacted patient responses to our interview questions.

In summary, this study demonstrates most patients are satisfied with their physicians' computer use during clinic visits and patients report EMR use has the potential to promote education and enhance communication. Patients reported negative perceptions related to physicians" "physical focus" (i.e., poor eye contact, lack of screen sharing, etc.) when using the EMR. Physicians of all levels should be taught patientcentered EMR use skills to enhance the patient-doctor relationship and communication in the digital age. Redesign of required EMR training in health care settings should incorporate these findings and include communication skills training.

Contributors: We acknowledge the following contributors: Julie Oyler $M D$, Lisa Vinci MD MS, Lynda Hale, the University of Chicago Section of General Internal Medicine, the University of Chicago Internal Medicine Residency Program and the University of Chicago Primary Care Group.

Prior Presentations: This research was presented as posters at the 2015 Society of General Internal Medicine (SGIM) Annual Meeting in Toronto, Canada in April 2015, the 2015 Association of American Medical Colleges (AAMC) Integrating Quality: Improving Value through Clinical Transformation, Education and Science Meeting in Chicago IL in June 2015, the 2015 AAMC Medical Education Meeting in Baltimore, MD in November 2015 and as an oral plenary presentation at the 2015 Midwest SGIM meeting in Cleveland OH in August 2015.

Corresponding Author: Wei Wei Lee, MD, MPH; Department of MedicineUniversity of Chicago, 5841 S. Maryland Avenue MC 3051, L325B, Chicago, IL 60637, USA (e-mail: wlee6@uchicago.edu).

\section{Compliance with Ethical Standards:}

Conflicts of Interest: The authors declare that they do not have a conflict of interest.

Funding: We were awarded a Picker Institute-Gold Foundation Challenge Grant for Residency Training, a University of Chicago Bucksbaum Institute Pilot Grant, and a grant from the Arnold P. Gold Foundation Research Institute Call for Reviews of Research on Humanistic Healthcare.

\section{REFERENCES}

1. Blumenthal D. Launching HITECH. N Engl J Med. 2010;362:382-38.

2. Schoen C, Osborn $\mathbf{R}$, Squires $\mathbf{D}$, et al. A survey of primary care doctors in ten countries shows progress in use of health information technology, less in other areas. Health Aff. 2012;31:2805-2816.

3. Irwin RS, Richardson ND. Patient-focused care, using the right tools. Chest. 2006; 130:73S-82S.

4. Matusitz J, Spear J. Effective doctor-patient communication: an updated examination. Soc Work Public Health. 2014;29(3):252-66.

5. Stewart MA. Effective physician-patient communication and health outcomes: a review. CMAJ Can Med Assoc J. 1995;152(9):1423.

6. Verghese A. Culture shock- patient as icon, icon as patient. N Engl J Med. 2008;359:2748-2751.

7. Alkureishi MA, Lee WW, Lyons MD, Press VG, Imam S, NkansahAmankra A, Werner D, Arora VM. Impact of electronic medical record use on the patient-doctor relationship and communication: a systematic review. J Gen Intern Med. 2015;31(5):548-60.

8. Makoul G, Curry RH, Tang PC. The use of electronic medical records: communication patterns in outpatient encounters. J Am Med Inform Assoc. 2001;8(6):610-5.

9. Booth N, Robinson P, Kohannejad J. Identification of high-quality consultation practice in primary care: the effects of computer use on doctor-patient rapport. Inform Prim Care. 2004;12(2):75-83.

10. Arar NH, Wen L, McGrath J, et al. Communicating about medications during primary care outpatient visits: the role of electronic medical records. Inform Prim Care. 2005; 13(1):13-22.

11. Johnson KB, Serwint JR, Fagan LA, et al. Computer-based documentation: effects on parent-provider communication during pediatric health maintenance encounters. Pediatrics. 2008;122(3):590-8.

12. Montague E, Asan O. Considering social ergonomics: the effects of HIT on interpersonal relationships between patients and clinicians. Work. 2012;41(Suppl 1):4479-83.

13. Als AB. The desk-top computer as a magic box: patterns of behaviour connected with the desk-top computer; GPs' and patients' perceptions. Fam Pract. 1997; 14(1):17-23.

14. Greatbatch D, Heath C, Campion P, et al. How do desk-top computers affect the doctor-patient interaction? Fam Pract. 1995; 12(1):32-6.

15. Dowell A, Stubbe M, Scott-Dowell $\mathbf{K}$, et al. Talking with the alien: interaction with computers in the GP consultation. Aust J Prim Health. 2013;19(4):275-82.

16. Shachak A, Hadas-Dayagi M, Ziv A, et al. Primary care physicians' use of an electronic medical record system: a cognitive task analysis. J Gen Intern Med. 2009;24(3):341-8.

17. Rouf E, Whittle J, Lu N, Schwartz MD. Computers in the exam room: differences in physician-patient interaction may be due to physician experience. J Gen Intern Med. 2007;22(1):43-8.

18. Street RL Jr, Liu L, Farber NJ, Chen Y, Calvitti A, Zuest D, Gabuzda MT, et al. Provider interaction with the electronic health record: the effects on patient-centered communication in medical encounters. Patient Educ Couns. 2014;96(3):315-9.

19. Fairley CK, Vodstrcil LA, Huffam S, et al. Evaluation of electronic medical record (EMR) at large urban primary care sexual health centre. PLoS One. 2013;Apr 4;8(4):e60636.

20. Furness ND, Bradford OJ, Paterson MP. Tablets in trauma: using mobile computing platforms to improve patient understanding and experience. Orthopedics. 2013;36(3):205-8.

21. Al Jafar E. Exploring patient satisfaction before and after electronic health record (EHR) implementation: the Kuwait experience. Perspect Health Inf Manag. 2013;Apr 1;10:1c

22. Jarvis B, Johnson T, Butler P, et al. Assessing the impact of electronic health records as an enabler of hospital quality and patient satisfaction. Acad Med. 2013;88(10):1471-7.

23. Ratanawongsa N, Barton JL, Schillinger D, et al. Ethnically diverse patients' perceptions of clinician computer use in a safety-net clinic. J Health Care Poor Underserved. 2013;24(4):1542-51.

24. Bushe GR. Advances in appreciative inquiry as an organization development intervention. Organ Dev J. 1999;17(2):61.

25. Gremler DD. The critical incident technique in service research. J Serv Res. 2004;7(1):65-89.

26. Pincavage AT, Lee WW, Beiting KJ, Arora VM. What do patients think about year-end resident continuity clinic handoffs? A qualitative study. J Gen Intern Med. 2013;28(8):999-1007.

27. Boeije H. A purposeful approach to the constant comparative method in the analysis of qualitative interviews. Qual Quant. 2002;36:339240 
28. Hruschka DJ, Schwartz D, St. John DC, Picone-Decaro E, et al. Reliability in coding open-ended data: lessons learned from HIV behavioral research. Field Method. 2004;16(3):307-331.

29. O'Brien BC, Harris IB, Beckman TJ, Reed DA, Cook DA. Standards for reporting qualitative research: a synthesis of recommendations. Acad Med. 2014;89(9):1245-1251.

30. Kumarapeli $\mathbf{P}$, de Lusignan $\mathbf{S}$. Using the computer in the clinical consultation; setting the stage, reviewing, recording, and taking actions: multi-channel video study. J Am Med Inform Assoc. 2013;20(e1):e67-75.

31. Garrison GM, Bernard ME, Rasmussen NH. 21st-century health care: the effect of computer use by physicians on patient satisfaction at a family medicine clinic. Fam Med. 2002;34(5):362-8.

32. Lee WW, Alkureishi ML, Farnan J, Arora V. Breaking Away from the iPatient to Care for the Real Patient: Implementing a Patient-Centered EMR
Use Curriculum. MedEdPORTAL: 2014. (accessed 5/26/2016) Available at: https://www.mededportal.org/publication/9953

33. Duke P, Frankel RM, Reis R. How to integrate the electronic health record and patient-centered communication into the medical visit: a skills based approach. Teach Learn Med. 2013;25(4):358-65.

34. Hsu $\mathbf{J}$, Huang $\mathbf{J}$, Fung $\mathbf{v}$, et al. Health information technology and physician-patient interactions: impact of computers on communication during outpatient primary care visits. J Am Med Inform Assoc. 2005; 12(4):474-480.

35. Pageler NM, Friedman CP, Longhurst CA. Refocusing medical education in the EMR Era. JAMA. 2013;310(21):2249-50.

36. Wald HS, George P, Rei, Taylor JS. Electronic health record training in undergraduate medical education: bridging theory to practice with curricula for empowering patient-and relationship-centered care in the computerized setting. Acad Med. 2014Mar;89(3):380-6. 